The third generation of gravitational wave observatories and their science reach

This article has been downloaded from IOPscience. Please scroll down to see the full text article.

2010 Class. Quantum Grav. 27084007

(http://iopscience.iop.org/0264-9381/27/8/084007)

View the table of contents for this issue, or go to the journal homepage for more

Download details:

IP Address: 194.94.224.254

The article was downloaded on 30/08/2012 at 10:50

Please note that terms and conditions apply. 


\title{
The third generation of gravitational wave observatories and their science reach
}

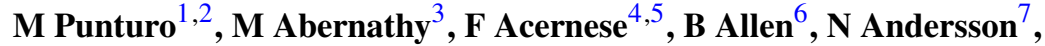

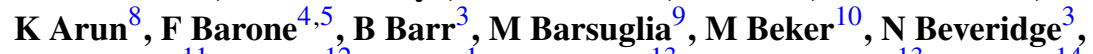 \\ S Birindelli $^{11}$, S Bose ${ }^{12}$, L Bosi $^{1}$, S Braccini ${ }^{13}$, C Bradaschia $^{13}$, T Bulik ${ }^{14}$, \\ E Calloni $^{4,15}$, G Cella $^{13}$, E Chassande Mottin ${ }^{9}$, S Chelkowski $^{16}$, \\ A Chincarini $^{17}$, J Clark ${ }^{18}, \mathbf{E}$ Coccia $^{19,20}, \mathbf{C ~ C o l a c i n o ~}^{13}, \mathbf{J ~ C o l a s ~}^{2}$, \\ A Cumming $^{3}$, L Cunningham ${ }^{3}$, E Cuoco $^{2}$, S Danilishin $^{21}$, K Danzmann ${ }^{6}$,

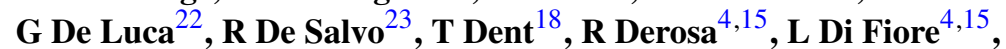 \\ A Di Virgilio ${ }^{13}$, M Doets ${ }^{10}$, V Fafone ${ }^{19,20}$, P Falferi $^{24}, \mathbf{R}$ Flaminio $^{25}$, \\ J Franc $^{25}$, F Frasconi ${ }^{13}$, A Freise ${ }^{16}$, P Fulda ${ }^{16}$, J Gair ${ }^{26}$, G Gemme ${ }^{17}$, \\ A Gennai $^{16}$, A Giazotto $^{2,13}$, K Glampedakis ${ }^{27}$, M Granata ${ }^{9}$, H Grote ${ }^{6}$,

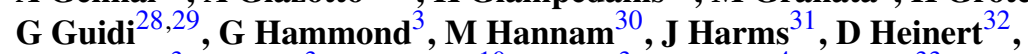

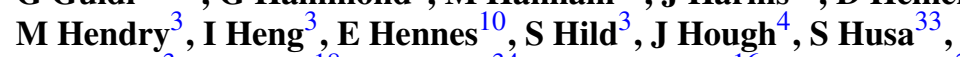 \\ S Huttner ${ }^{3}$, G Jones ${ }^{18}$, F Khalili ${ }^{34}$, K Kokeyama ${ }^{16}$, K Kokkotas ${ }^{27}$,

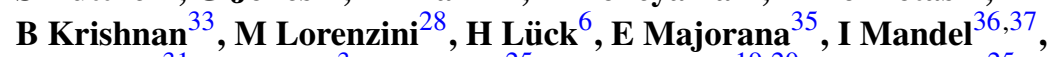 \\ V Mandic $^{31}$, I Martin $^{3}, \mathbf{C ~ M i c h e l}^{25}, \mathbf{Y}$ Minenkov ${ }^{19,20}, \mathbf{N}$ Morgado $^{25}$, \\ S Mosca ${ }^{4,15}$, B Mours ${ }^{38}$, H Müller-Ebhardt ${ }^{6}$, P Murray $^{3}$, R Nawrodt $^{3}$, \\ J Nelson $^{3}$, R Oshaughnessy ${ }^{39}$, C D Ott $^{40}$, C Palomba $^{35}$, A Paoli $^{2}$, \\ G Parguez $^{2}$, A Pasqualetti ${ }^{2}$, R Passaquieti ${ }^{13,41}$, D Passuello ${ }^{13}$, \\ L Pinard $^{25}$, R Poggiani ${ }^{13,41}$, P Popolizio ${ }^{2}$, M Prato ${ }^{17}$, P Puppo $^{35}$, \\ D Rabeling ${ }^{10}$, P Rapagnani ${ }^{35,42}$, J Read ${ }^{33}$, T Regimbau ${ }^{11}, \mathbf{H} \operatorname{Rehbein}^{6}$,

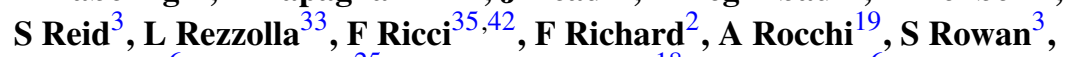 \\ A Rüdiger $^{6}$, B Sassolas $^{25}$, B Sathyaprakash $^{18}$, R Schnabel $^{6}$,

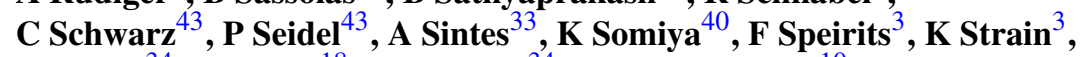

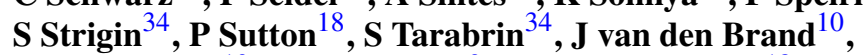 \\ $\mathbf{C}$ van Leewen ${ }^{10}, \mathbf{M}$ van Veggel $^{3}, \mathbf{C}$ van den Broeck ${ }^{18}$, A Vecchio ${ }^{16}$,

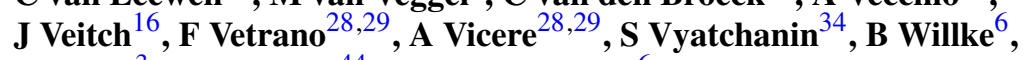 \\ G Woan $^{3}$, P Wolfango ${ }^{44}$ and K Yamamoto ${ }^{6}$ \\ ${ }^{1}$ INFN, Sezione di Perugia, I-6123 Perugia, Italy \\ 2 European Gravitational Observatory (EGO), I-56021 Cascina (Pi), Italy \\ ${ }^{3}$ Department of Physics and Astronomy, The University of Glasgow, Glasgow, G12 8QQ, UK \\ ${ }^{4}$ INFN, Sezione di Napoli, Italy \\ ${ }^{5}$ Università di Salerno, Fisciano, I-84084 Salerno, Italy \\ ${ }^{6}$ Max-Planck-Institut für Gravitationsphysik, D-30167 Hannover, Germany \\ ${ }^{7}$ University of Southampton, Southampton s0171BJ, UK \\ 8 LAL, Université Paris-Sud, IN2P3/CNRS, F-91898 Orsay, France \\ 9 AstroParticule et Cosmologie (APC), CNRS; Observatoire de Paris-Université Denis \\ Diderot-Paris VII, France \\ 10 VU University Amsterdam, De Boelelaan 1081, 1081 HV, Amsterdam, The Netherlands \\ 11 Université Nice-Sophia-Antipolis, CNRS, Observatoire de la Côte d'Azur, F-06304 Nice, \\ France \\ 12 Washington State University, Pullman, WA 99164, USA \\ ${ }^{13}$ INFN, Sezione di Pisa, Italy
}


14 Astro. Obs. Warsaw Univ. 00-478; CAMK-PAM 00-716 Warsaw; Bialystok Univ. 15-424; IPJ 05-400 Swierk-Otwock; Inst. of Astronomy 65-265 Zielona Gora, Poland

15 Universitá di Napoli 'Federico II 'Complesso Universitario di Monte S. Angelo', 1-80126 Napoli, Italy

16 University of Birmingham, Birmingham, B15 2TT, UK

17 INFN, Sezione di Genova, I-16146 Genova, Italy

${ }^{18}$ Cardiff University, Cardiff, CF24 3AA, UK

19 INFN, Sezione di Roma Tor Vergata 1-00133 Roma, Italy

${ }^{20}$ Universitá di Roma Tor Vergata, I-00133, Roma, Italy

21 Moscow State University, Moscow, 119992, Russia

22 INFN, Laboratori Nazionali del Gran Sasso, Assergi l'Aquila, Italy

${ }^{23}$ LIGO, California Institute of Technology, Pasadena, CA 91125, USA

${ }^{24}$ INFN, Gruppo Collegato di Trento, Sezione di Padova; Istituto di Fotonica e Nanotecnologie, CNR-Fondazione Bruno Kessler, 38123 Povo, Trento, Italy

${ }^{25}$ Laboratoire des Matériaux Avancés (LMA), IN2P3/CNRS, F-69622 Villeurbanne, Lyon, France

${ }^{26}$ University of Cambridge, Madingley Road, Cambridge, CB3 OHA, UK

27 Theoretical Astrophysics (TAT) Eberhard Karls University of Tuebingen Auf der Morgenstelle 10, 72076 Tübingen, Germany

28 INFN, Sezione di Firenze, I-50019 Sesto Fiorentino, Italy

${ }^{29}$ Università degli Studi di Urbino 'Carlo Bo', I-61029 Urbino, Italy

${ }^{30}$ Department of Physics, University of Vienna, Boltzmanngass 5, A-1090 Vienna, Austria

${ }^{31}$ University of Minnesota, Minneapolis, MN 55455, USA

32 Friedrich-Schiller-Universität Jena PF 07737 Jena, Germany

33 Max Planck Institute for Gravitational Physics (Albert Einstein Institute) Am Mühlenberg 1, D-14476 Potsdam, Germany

${ }^{34}$ Moscow State University, Moscow, 119992, Russia

35 INFN, Sezione di Roma 1, 1-00185 Roma, Italy

36 Department of Physics and Astronomy, Northwestern University, Evanston, IL 60208, USA

37 NSF Astronomy and Astrophysics Postdoctoral Fellow

38 LAPP-IN2P3/CNRS, Université de Savoie, F-74941 Annecy-le-Vieux, France

39 The Pennsylvania State University, University Park, PA 16802, USA

${ }^{40}$ Caltech-CaRT, Pasadena, CA 91125, USA

${ }^{41}$ Università di Pisa, I-56127 Pisa, Italy

42 Università 'La Sapienza', I-00185 Roma, Italy

43 INFN, Sezione di Roma Tre and Universitá di Roma Tre-Dipartimento di Fisica, I-00146 Roma, Italy

${ }^{44}$ Università degli Studi di Firenze, I-50121, Firenze, Italy

E-mail: michele.punturo@pg.infn.it

Received 12 November 2009, in final form 12 January 2010

Published 6 April 2010

Online at stacks.iop.org/CQG/27/084007

\section{Abstract}

Large gravitational wave interferometric detectors, like Virgo and LIGO, demonstrated the capability to reach their design sensitivity, but to transform these machines into an effective observational instrument for gravitational wave astronomy a large improvement in sensitivity is required. Advanced detectors in the near future and third-generation observatories in more than one decade will open the possibility to perform gravitational wave astronomical observations from the Earth. An overview of the possible science reaches and the technological progress needed to realize a third-generation observatory are discussed in this paper. The status of the project Einstein Telescope (ET), a design study of a third-generation gravitational wave observatory, will be reported. 
PACS number: $04.80 . \mathrm{Nn}$

(Some figures in this article are in colour only in the electronic version)

\section{Introduction}

The first generation of interferometric gravitational wave (GW) detectors (GEO600 [1], LIGO [2], TAMA [3], Virgo [4]) approximately reached their design sensitivities and thus demonstrated the effectiveness of the working principle. The major detectors currently operative are an enhanced version of the first generation (Virgo+ and E-LIGO), with larger laser power and some technological improvements.

Advanced detectors (like 'Advanced LIGO' [5] and 'Advanced Virgo' [6]) will show a sensitivity improved roughly by a factor of 10 with respect to the initial interferometers. They are based on technologies currently available, sometimes tested in reduced scale prototypes, but still to be implemented in full scale. According to the current models of GW sources, sensitivity of the advanced interferometers is expected to guarantee the detection of the signals generated by astro-physical sources within months to a year at most. For example, at the nominal sensitivity of the advanced detectors, the expected detection rate of the GW signal generated by a binary system of coalescing neutron stars is about a few tens per year [7,8]. But apart from extremely rare events, the expected signal-to-noise ratio (SNR) of these detections obtained with the advanced detectors is too low for precise astronomical studies of the GW sources and for complementing optical and x-ray observations in the study of fundamental systems and processes in the universe.

These considerations led the GW community to start investigating a new (third) generation of detectors. With considerably improved sensitivity, these new machines will open the era of routine GW astronomy. Since the first detection of a GW signal is expected to occur in the advanced interferometers, it is especially the understanding and enhancement of the observational aspects that the planning of the third generation must focus on.

To realize a third-generation GW observatory with a significantly enhanced sensitivity (let us consider a target of a factor of 10 improvement in sensitivity with respect to the advanced detectors in a wide frequency range), several limitations of the technologies adopted in the advanced interferometers must be overcome and new solutions must be developed to reduce the fundamental and technical noises that will limit the next-generation machines.

In this paper, for lack of space availability, we will focus on a small subset of the possible scientific goals of a third-generation GW observatory and give a short overview of the technological challenges introduced by this new generation of machines and under evaluation within the framework of the Einstein Telescope (ET) design study [9].

\section{Science reaches}

Figure 1 shows the typical expected sensitivity and the main noise sources of an advanced gravitational wave detector. If a third generation gravitational wave observatory is realized with a sensitivity of about a factor 10 more with respect to an advanced interferometer, as shown in figure 2 [10], it will possible to open a new avenue for understanding physics of the extreme phenomena in the universe $[11,12]$. 


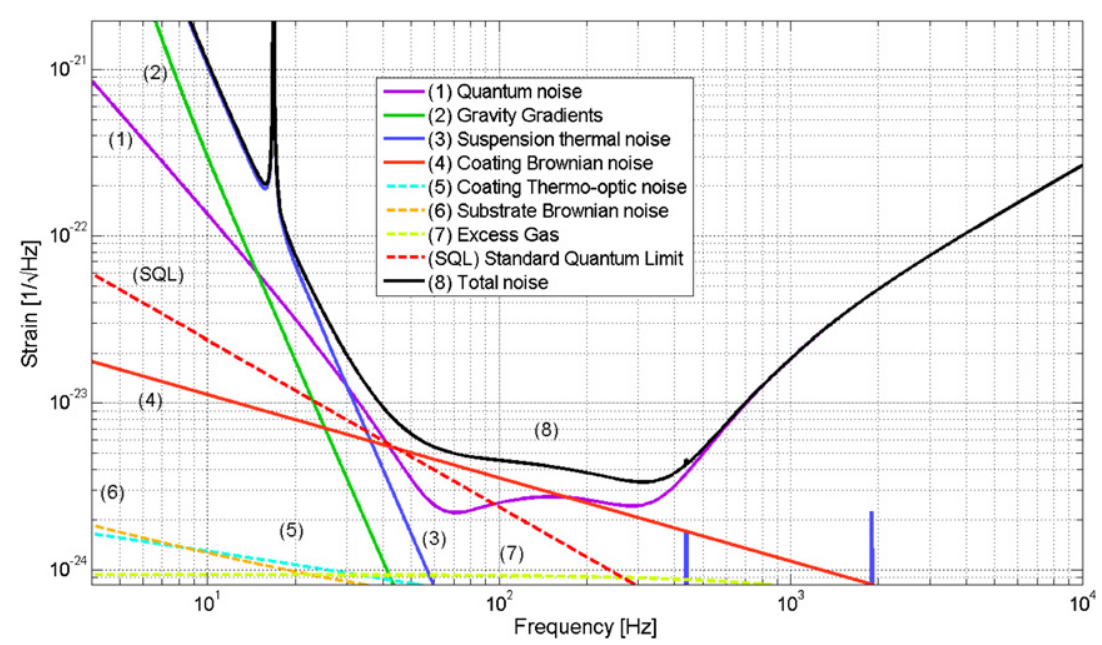

Figure 1. Typical sensitivity of a second-generation GW detector shown here for advanced Virgo with $125 \mathrm{~W}$ of injected power. The sensitivity is dominated at very low frequency by the seismic noise, at low and intermediate frequencies by the suspension and mirror thermal noises and radiation pressure noise and at high frequencies by the shot noise.

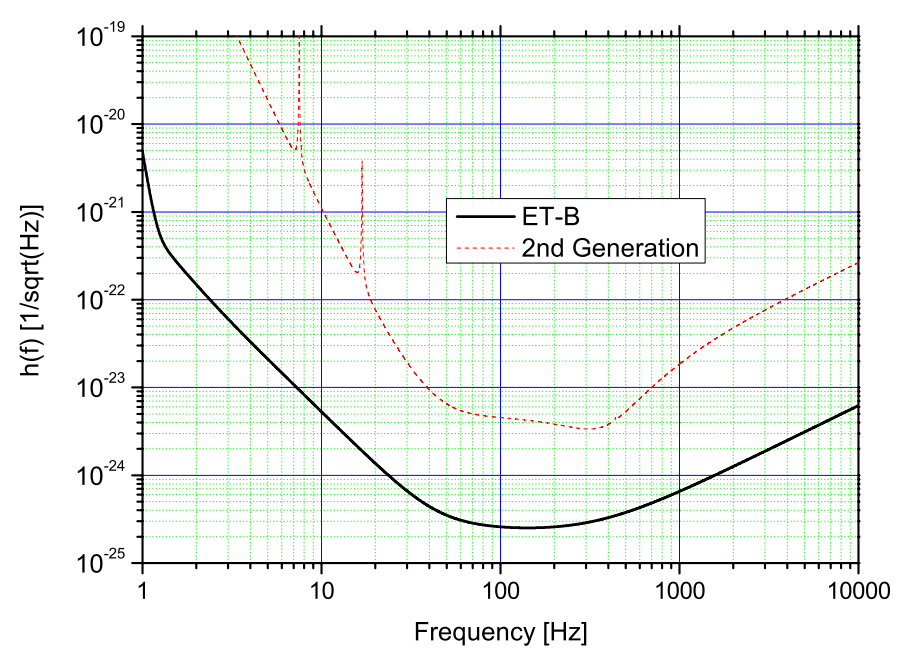

Figure 2. A possible sensitivity (tick curve) of an underground, long suspension, cryogenic, signal and power recycled single third-generation gravitational wave observatory (see table 1 in [10]) compared to a typical sensitivity curve of an advanced detector (dashed curve). It is worth to underline that the evaluation of the possible noise level of a third-generation GW observatory is an ongoing activity, still far to be concluded within the ET design study. For these reasons, the curves are updated regularly and labeled with progressive letters to be distinguished. In the tick curve (so-called ET-B), corresponding to a single wide-band detector, the suspension thermal noise contribution is missing.

Astrophysics: Measure in great detail the physical parameters of compact stars (i.e. neutron stars (NS) and black holes (BH)) in a binary system [13, 14], constrain the equation-of-state of NS and solve the enigma of gamma ray bursts (GRB) [11, 15]. 

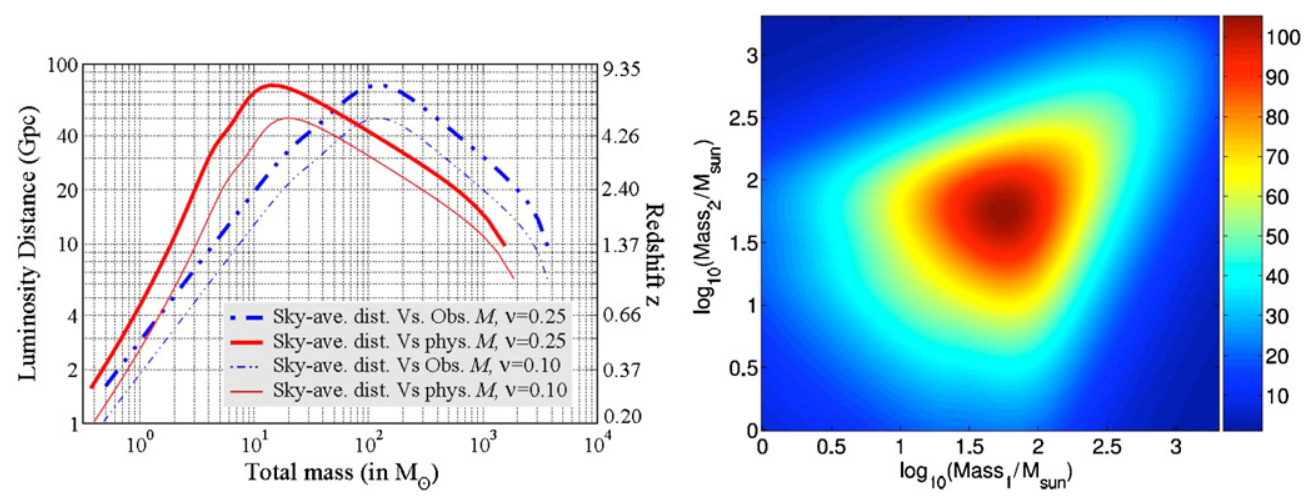

Figure 3. Left: the distance reach of ET-B (at an SNR $=10$ ) for inspiral signals as a function of the intrinsic ((red) solid line) and observed ((blue) dashed line) total mass. Right: SNR for binaries at a distance of $3 \mathrm{Gpc}$ as a function of the component masses.

General relativity: Test general relativity by comparing observations of massive binary star systems with numerical relativity (NR) predictions and constrain alternative theories of gravity (such as the Brans-Dicke theory) through the observation of NS-BH coalescences [11].

Cosmology: Measure cosmological parameters from standard sirens of gravity [16, 17] and probe the primordial universe through the measurement of the GW stochastic background [11].

Astroparticle physics: Measure or constrain the neutrino and graviton masses through the detection of the GW emitted in a supernova [18].

In what follows we will describe a few selected topics; a more detailed description can be found in the ET science vision document [11].

\subsection{Binary systems}

Binary neutron stars (NS-NS) could be seen up to a distance of $400 \mathrm{Mpc}$ in advanced detectors. The expected rate of mergers within that distance gives an event rate of about 40 per year [7] with guaranteed detections. The low signal-to-noise ratio (SNR) of such events might not permit detailed measurement of the physical properties of the sources but advanced detectors could begin to impact cosmography by independently measuring the Hubble constant to a few percent [19]. This is because binary neutron stars are self-calibrating standard sirens, allowing a direct measurement of their luminosity distance and sky location from a knowledge of the gravitational-wave amplitude in a network of three or more detectors. The total mass, mass ratio and other intrinsic parameters of the binary can all be measured, by matched filtering the data with templates that have been computed to a very high accuracy [20-23] using approximation methods to solve Einstein's equations. This would be a milestone for cosmology, especially since there is currently a gap in the measurement of the expansion rate of the universe at around $300 \mathrm{Mpc}$ (see e.g. [24], especially their figure 1 where one can see that measurements are sparse in the red-shift range $0.1<z<0.4$ ).

The expected distance up to which the inspiral phase of compact binaries could be detected is shown in figure 3 (left panel) which reveals that binary neutron stars can be observed from a red-shift of $z \sim 2$. One should expect to detect millions of binary neutron star mergers per year within the distance reach of ET. Red-shift could be measured to a small fraction 
Table 1. Errors in the determination of the cosmological parameters through the detection of 5192 realizations of a catalogue containing 1000 BNS merger events, of known red-shift [17]. The fractional $1-\sigma$ widths of the distributions $\sigma_{\Omega_{\Lambda}} / \Omega_{\Lambda}, \sigma_{\Omega_{M}} / \Omega_{M}$ and $\sigma_{w} /|w|$ are shown, accounting for the weak lensing errors in the left column and considering it corrected in the right column. The meaning of these parameters is described in [17] and briefly hereafter: $\Omega_{M}$ and $\Omega_{\Lambda}$ are the (dimensionless) energy densities of the dark matter and dark energy, respectively; $w$ is the dark energy equation of state parameter ( $w=1$ corresponds to a cosmological constant).

\begin{tabular}{lcccccc}
\hline Free parameters & \multicolumn{2}{c}{$\sigma_{\Omega_{\Lambda}} / \Omega_{\Lambda}$} & \multicolumn{2}{c}{$\sigma_{\Omega_{M}} / \Omega_{M}$} & \multicolumn{2}{c}{$\sigma_{w} /|w|$} \\
\hline 3 & $4.2 \%$ & $3.5 \%$ & $18 \%$ & $14 \%$ & $18 \%$ & $15 \%$ \\
2 & $\Omega_{\Lambda}=0.73$ & $9.4 \%$ & $8.1 \%$ & $7.6 \%$ & $6.6 \%$ \\
1 & $\Omega_{\Lambda}=0.73$ & $\Omega_{M}=0.27$ & $1.4 \%$ & $1.1 \%$ \\
\hline
\end{tabular}

(say, 1 in 1000) of these events, for instance by identifying them in coincidence with gammaray bursts. The measured values of red-shift and luminosity distance can be fitted to a cosmological model to infer cosmological parameters. Expected errors in the estimation of the cosmological parameters shown in table 1 [17] are comparable to those from other cosmology missions such as the Joint Dark Energy Mission.

The distance reach of ET shown in figure 3 is computed using signals in the so-called restricted post-Newtonian $(\mathrm{PN})$ approximation. This is an approximation in which one retains the amplitude of the waveform to the lowest order in PN expansion neglecting higher order corrections. At the lowest order, the signal contains only the second harmonic of the orbital frequency while the higher-order corrections contain other harmonics. The effects of subdominant harmonics are not completely understood yet but we already know that they could facilitate observation of heavier binaries as well as greatly improve the accuracy with which the source parameters can be measured [13, 14, 25]. Accurate measurement of the parameters will allow the determination of the mass function of neutron stars and black holes, the maximum mass of a neutron star and its equation-of-state and provide a comprehensive history of the formation and evolution of compact binaries. Such studies will give answers to other astrophysical questions such as the history of star formation, the birth of intermediate-mass black holes and their growth [26, 27], etc.

Observation of the merger of binary black holes can open a new era for fundamental physics. Moments before the coalescence, the component black holes in a binary experience ultrastrong gravitational fields created by each other. Recent advances in analytical [28-34] and numerical relativity [35-41] has made it possible to predict the characteristics of the signal emitted in the process of merger. Comparison of the predicted signal amplitudes and fluxes with the observation will enable tests of general relativity that can never be carried out by any other means. It should be possible to check if the spacetime geometry of the merged object is that of a black hole or some other exotic object, if black holes are enclosed in a horizon, etc.

\subsection{Isolated neutron stars}

Neutron stars (NS) are one possible end state of massive stars that result in a gravitational collapse followed by supernova of type II, type Ib or type Ic. Our knowledge of neutron stars comes mainly from the observation of the radio and x-ray emissions of hundreds of pulsars currently known. The composition of a NS and the cause of its pulsation in radio are still under debate. It is believed that the NS crust consists of ordinary matter (chiefly iron) ordered in an atomic lattice embedded in a sea of free electrons. The outer core could consist of a mixture of nuclei, free protons, neutrons and electrons. The inner core of the star could be a 

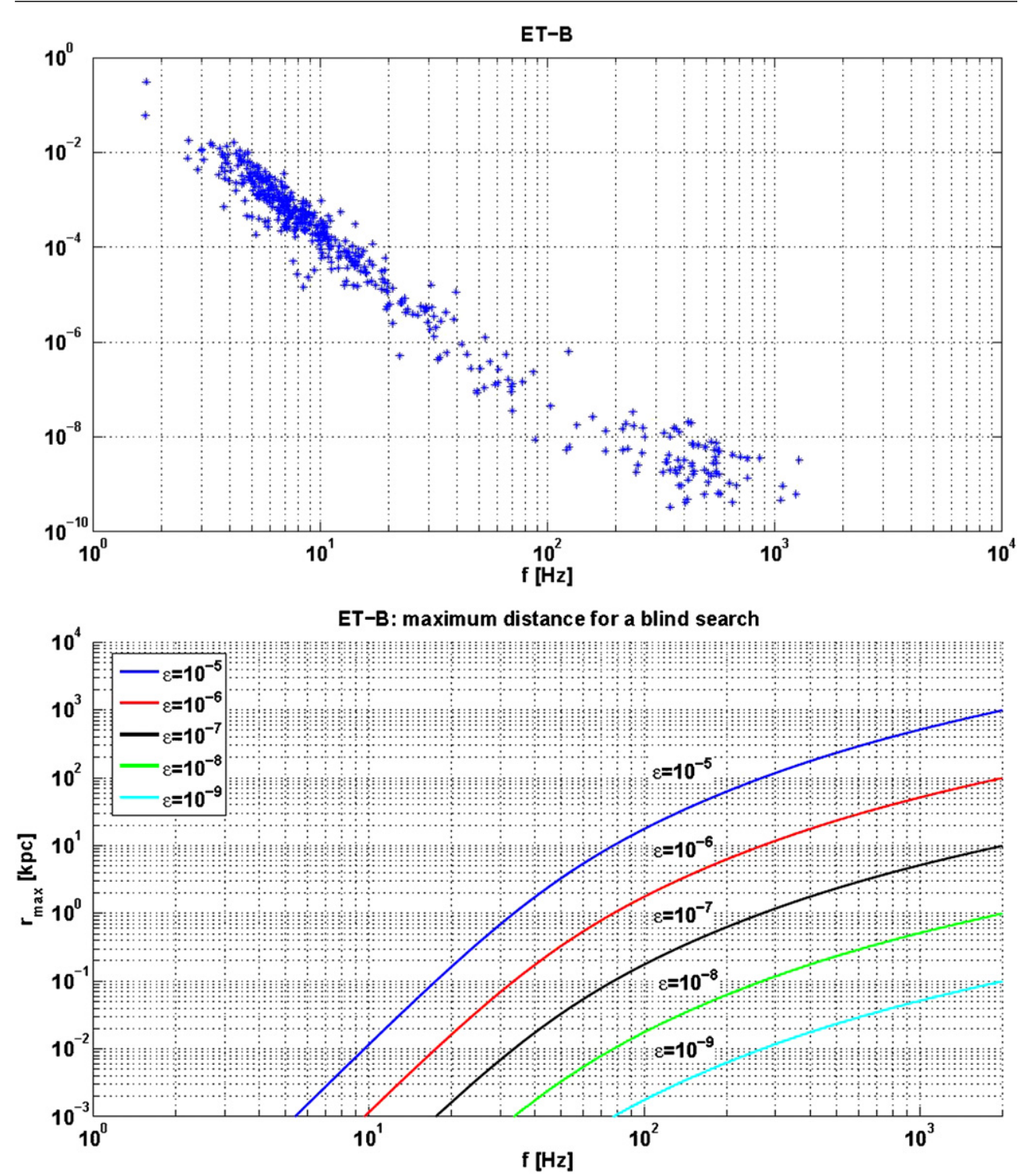

Figure 4. Upper panel: smallest ellipticity of known pulsars detectable in ET (ET-B sensitivity) [11]. Lower panel: the detection range for a blind search of NS in ET (ET-B sensitivity) [11]. The uppermost curve corresponds to an ellipticity $\epsilon=10^{-5}$ and the lowest to $\epsilon=10^{-9}$.

superfluid of neutrons. According to some models, the core could consist of strange quarks (see [42] for a review).

The main mechanism for the emission of GW by a NS is due to the quadrupolar moment generated by its ellipticity $\epsilon$. In this case, the GW emission occurs at twice the rotational frequency of the NS. The amount of ellipticity $\epsilon$ that a NS could support is related to the equation-of-state of the ultra-dense nuclear matter of the star. According to the current models the crust of the star could support $\epsilon \simeq 10^{-6}-10^{-7}$, whereas the solid core could sustain up to $\epsilon \simeq 10^{-3}$. A higher ellipticity could indicate a solid quark star. Figure 4, upper panel, shows the minimum ellipticity in known pulsars that would be detectable in 'ET-B' (see 
figure 2 [10]). The lower panel shows the detection range, for different ellipticities, for a blind search (i.e. unknown sky position and frequency). The possibility of detecting continuous GW emission of NS having ellipticity below $10^{-6}-10^{-7}$ will place interesting constrains on the supra-nuclear equation-of-state.

Just as helioseismology helped determine the internal structure of the Sun, asteroseismology — the study stellar oscillations - could help constrain the equation-of-state of a NS. Glitches in pulsars are believed to be the result of stellar quakes that could excite quasi-normal mode oscillations of the star, emitting gravitational waves with characteristic frequencies and damping times [43]. By observing the frequency and damping times of the quasi-normal modes, it should be possible to measure the mass and radius of a neutron star with an accuracy of $10 \%$ or better [44], which would constrain the equation-of-state of the star and hence its composition.

\subsection{Supernova explosion and neutrino mass measurement}

A supernova ( $\mathrm{SNe}$ ) caused by the collapse of a massive star's iron core to a NS is the most energetic event in the universe, releasing $\sim 10^{53}$ erg of gravitational energy. Most of this energy $(\sim 99 \%)$ is emitted in neutrinos, but a very small fraction, depending on the asymmetry of the collapse, could be emitted in GW. The precise mechanism of the collapse and the ensuing supernova is still unknown and, hence, the amount of energy emitted in GW is under debate, but recent evaluations (see [45] for a review) limit it to $10^{-7}-10^{-8}$ solar masses. The possibility of coincident measurement of neutrinos, electromagnetic waves and gravitational waves could put a very strong constraint on the core collapse supernova mechanism. The limited amount of energy emitted in GW (consequently, the reduced distance reach) and the low SNe rate in the Milky Way and Local Group (less than 1 event per two decades [46]) make the detection of the GW emitted by SNe rather improbable in initial and advanced detectors. Einstein Telescope might be sensitive to supernovae at a few $\mathrm{Mpc}$, with an expected event rate of $\sim 0.5$ year $^{-1}$ [47]. If, as expected, at the time of the implementation of the ET observatory, megaton-class neutrino detectors are operational and have a range similar to ET, it will be possible to provide coincident observations with an acceptable event rate.

The (almost) simultaneous detection of GW and neutrinos could result in an independent measurement of the neutrino mass. In fact, the time delay between the two detections can be expressed as $\Delta t=\Delta t_{S N}+\Delta t_{p}+\Delta t_{d}$, where $\Delta t_{S N}$ is the emission time difference, $\Delta t_{p}$ is the propagation time and $\Delta t_{d}$ is the time difference due to the different location of the detectors. If $\Delta t_{S N}$ is known through the model (or if it is lower than $1 \mathrm{~ms}$ ), it is possible to measure the neutrino mass with an accuracy better than $1 \mathrm{eV}$ [18].

\section{Limits of the second-generation detectors and technological challenges for the third generation}

The typical sensitivity of an advanced or second-generation interferometric gravitational wave detector is shown in figure 1 . If we consider only the fundamental noises, the sensitivity of these apparatuses will be dominated

- at very low frequency (below 4-5 Hz) by the seismic noise and the gravity gradient noise (see section 3.1),

- in the 4-50 Hz range by the thermal noise (see section 3.2.1) of the optics suspension system and by the quantum noise, related to the radiation pressure (see section 3.3.5) exerted on the suspended mirror by the photons in the main Fabry-Perot cavities, 
- in the 40-300 $\mathrm{Hz}$ range by the thermal noise of the suspended mirrors (mainly the coating contribution, see section 3.2.2) and

- at higher frequencies by the shot noise component of the quantum noise (see section 3.3.2).

\subsection{Seismic noise}

Ground based interferometric GW detectors are and will be limited, in the very low frequency range, by the natural and anthropomorphic vibration of the soil where the instrument is realized.

Seismic noise acts on the suspended test masses in two ways:

- through the suspension chain, shaking each stage according to its transfer function,

- coupling the mass vibration in the soil layers, perturbed by the seismic waves, directly with the test-mass displacement, via the mutual attraction force expressed by Newton's universal law of gravitation (so-called gravity gradient noise or Newtonian noise).

3.1.1. Seismic noise filtering. In a GW detector, the seismic vibrations must be sufficiently filtered by the suspension chain before it reaches the test masses. Currently the filtering system is realized through a chain of harmonic oscillators that filter the seismic vibration horizontally (inverted and 'normal' pendulums) and vertically (blades). Virgo currently implemented the most sophisticated of such passive filtering systems and it shows the best sensitivity below $40 \mathrm{~Hz}$. The so-called Virgo Super-Attenuator pushes the residual seismic noise below the thermal noise of a first-generation detector like Virgo starting from about $4 \mathrm{~Hz}$ [48]. Recent evaluations [49], supported by direct measurements in the Virgo detector, demonstrated the full validity of the passive filtering design also considering the stringent requirements of a second-generation GW detector like Advanced Virgo, where the suspension thermal noise is reduced by an order of magnitude.

Passive filtering is not the only way attempted to fight the seismic noise in a GW detector; in the advanced LIGO design, an active philosophy [50] has been implemented: in a chain of three sub-systems, the displacement and the accelerations caused by the seismic noise are read through position and acceleration sensors and are actively and hierarchically suppressed through hydraulic and electromagnetic actuators.

In a third-generation GW detector, there are additional requirements that make the achievement of the sensitivity targets very challenging; in fact, the first requirement is the improvement of the noise level in relation to the advanced detectors roughly by a factor of 10 in the whole detection frequency band, but the second and more difficult requirement is to access the frequency region between 1 and $10 \mathrm{~Hz}$, excluded in the advanced detectors. The achievement of both these targets is too challenging to be requested from an improved seismic filtering system and alternative (or better additive) solutions must be found. It is well known that underground sites are seismically quieter (i.e. see [51]) and the possibility of realizing an underground GW detector has been analyzed and selected by the LCGT [52] (Large Scale Cryogenic GW Telescope) collaboration in Japan. The comparison between the seismic noise in the TAMA [53] site (Tokyo) and in the LISM [54] site (Kamioka mine, the prime candidate as an LCGT site) shows a reduction in the low-frequency region, by going underground, by a factor of 100 in terms of acceleration and by two to three orders of magnitude in displacement spectral amplitude. A corresponding and even larger noise reduction has been reached in the output of the LISM interferometer, due to the fact that going underground several other 'technical' noises, induced by external disturbances like wind, scattered light or temperature fluctuations, are suppressed by the quietness of the site. 
Hence, an underground site can offer the possibility of achieving the desired sensitivity improvement with respect to an advanced detector in the same frequency band. A preliminary investigation [49] on the compliance of the Virgo Super-Attenuator with the requirements of a third-generation detector has shown a satisfactory behavior above $4 \mathrm{~Hz}$, whereas to access the 1-3 Hz frequency range, major technical upgrades of the suspension system must be realized.

3.1.2. Gravity gradient noise. In the GW interferometers, the suspended test masses are subject to the random gravitational forces generated by seismic noise and by moving massive bodies [55-57]. Obviously the importance of this disturbance depends on the seismic noise level and on the contribution of the other low-frequency noise sources to the noise budget; it is evident that the seismic filtering chain doesn't play any role for this noise source, since the gravitational force is a direct interaction between the suspended mirrors and the neighboring masses. In the first generation of GW detectors, the gravity gradient noise does not play any role and in the advanced detectors (due to the improvement of the sensitivity at low frequencies), it starts approaching the overall noise level, but remaining negligible above $10 \mathrm{~Hz}$ (see figure 1).

In the third generation of GW detectors, the more stringent requirements in terms of sensitivity at low frequency enhance the importance of this noise source. The reduction of the seismic noise, due to the possibility of realizing the observatory in an underground site, opens new questions about the validity of the current noise modeling. Recent evaluations $[58,59]$ have shown the importance, in the noise modeling, of the surface seismic waves, the irrelevance of the experimental cavity shape and the attenuation, going deeply underground ( $\gtrsim 100 \mathrm{~m}$ ), of the gravity gradient noise, at least above 1-2 Hz. If that very low frequency range needs to be accessed to fulfil the science requirements of a third-generation observatory, a major effort must be made to complement the seismic attenuation with the subtraction of the residual gravity gradient noise through the signals extracted from a network of sensors located around the detector [58].

\subsection{Thermal noise}

By thermal noise we indicate all those processes that modulate the optical path of the light in the interferometer coupling it to the Brownian fluctuation or to the stochastic fluctuation of the temperature field in the optical components. It is common to distinguish between the suspension thermal noise, affecting the position of the test masses (considered a rigid body) through the fluctuations of the suspension wires or fibers, and the mirror thermal noise, which is the overlap of all the fluctuation and dissipation processes occurring in the test masses and in the high reflectivity coatings.

3.2.1. Suspension thermal noise. To model and understand the thermal noise in the interferometers (in thermal equilibrium) two fundamental instruments are used: the equipartition theorem, which relates the temperature of a system to its average energies, and the fluctuation-dissipation theorem (FDT) [60], which relates the power spectrum of the fluctuations of a system (in thermal equilibrium) to its dissipation processes, described by the mechanical impedance. The equi-partition theorem tells how much thermal energy is present in a thermo-dynamical system, whereas the FDT describes how that energy is distributed in frequency. The strategies to reduce the thermal noise impact in the second-generation GW detectors are essentially an evolution of what has been applied in the initial machines and are essentially based on the reduction of the dissipation in the suspension system, in order to 
concentrate all the fluctuation energy into the normal modes of the system, resulting in a low noise level off-resonance.

Already in the initial detectors, particular attention has been devoted to the reduction of the dissipation in the suspension by selecting the right material for the suspension wires [61-64], by optimizing the clamping [65] and the mirror-to-wire contact [66] design. Thanks to the pioneering work of the Glasgow group in the GEO600 collaboration $[67,68]$, the second generation of GW detectors will adopt the so-called monolithic [69] suspensions (already operative in GEO600 since 2001), fully realized in fused silica, which simultaneously reduces the clamping losses and minimizes the losses in the suspension fibers, because of the low mechanical dissipation of fused silica [70].

In addition to the minimization of the mechanical losses through the selection of the best materials and geometries, another means for reducing the suspension thermal noise must be used in the third generation of GW detectors: the temperature. According to the equipartition theorem, the temperature is directly proportional to the energy stored in each degree of freedom of the suspended system allowing to reduce the fluctuation amplitude by lowering the temperature. Furthermore, at low temperature, some materials show a suppression of the dissipation mechanisms.

Hence, cryogenics is one of the most appealing technologies to reduce the thermal noise of the optics suspension in a third-generation GW detector. The first problem to be solved in a cryo-interferometer is how to cool down the test masses without introducing additional vibrations that spoil the very low frequency performances. New technologies are now available, for example based on cryo-cooling systems [71] that are actively damped to reduce the seismic vibration and that promise an easier achievement of low temperatures with less impact on the low-frequency performance of the interferometer than previous cryo-coolers. A special design of the suspension system is needed to cool down the test mass without introducing additional vibration. Progress in channeling the heat transmission through a second, parallel seismic filtering system has been made in the ILIAS [72] project.

Even more important is the right choice of the material for the realization of the last stage of the suspension system. The fused silica suspension, developed for the second-generation GW detectors, cannot be used in cryogenics because of its poor thermal conductivity and because of a well-known dissipation peak of that amorphous material at low temperature. In order to be a good candidate for a cryogenic suspension, the material must exhibit a high thermal conductivity at the operation temperature chosen, to permit an efficient heat extraction (which is crucial, because of the relatively high heating power deposited in the test masses by the high light power stored in the interferometer cavities), a low mechanical dissipation angle (to reduce the Brownian thermal noise), a low thermal expansion coefficient (to minimize the thermo-elastic noise) and a good breaking strength (to safely support the test masses). Currently there are two candidate materials for this role: sapphire and silicon. Sapphire has been selected to realize the suspension fibers of LCGT [52] both for its dissipation [73] and for its thermal conductivity properties [74]; silicon, on the other hand, has been preliminarily studied within the ILIAS project and it has been found suitable to realize both suspension fibers [75] and ribbons [76]. However, it is the matter of fact that currently only sapphire has been used to realize a full cryogenic suspension and the usage of silicon still needs a successful R\&D activity.

3.2.2. Mirror thermal noise. The major limitation of the sensitivity of the second generation of GW detectors in the $40-200 \mathrm{~Hz}$ range will be the thermal noise related to the high reflectivity dielectric coating applied to the main cavities mirrors. While the mirror substrate material chosen (synthetic fused silica) shows low mechanical dissipation, the high refraction 
component in the dielectric coating (tantalum pentoxide $\mathrm{Ta}_{2} \mathrm{O}_{5}$ ) shows high mechanical losses that dominate the dissipation of the test mass [77, 78].

Third-generation GW detectors could benefit from all the R\&D activities currently performed to reduce the mirror coatings thermal noise contribution in the advanced GW detectors (include titanium dopants in the tantalum layers [79], optimization of the amount of high dissipation material in the coating [80, 83]); but, unfortunately, we cannot expect a further reduction of the dissipation if a cryogenic-interferometer is realized (and tantalasilica-based coatings are used). In fact, measurements performed to characterize the coatings for the LCGT detectors have shown that the mechanical dissipation in a multi-layer tantalum pentoxide coating is rather independent of the temperature [81] and more recent measurements [82] have even shown a low-temperature dissipation peak in a single layer of $\mathrm{Ta}_{2} \mathrm{O}_{5}$ doped with $\mathrm{TiO}_{2}$.

Furthermore, because of the broad dissipation peak shown by fused silica at low temperature, in the cryo-interferometers it is impossible to choose the low mechanical loss, low optical absorption substrates developed for the advanced detectors. Hence, as a result from the suspension development (see section 3.2.1), currently the best material candidate to realize the test masses are sapphire (selected in LCGT) and crystalline silicon, showing a very low mechanical dissipation angle (about $3-4 \times 10^{-9}$ ) at low temperature. Sapphire is transparent to the standard wavelength adopted in the $\mathrm{GW}$ detectors $(1064 \mathrm{~nm})$. It shows relatively small thermal lensing [84] due to its large thermal conductivity at low temperature $\left(2330 \mathrm{~W} \mathrm{~m}^{-1} \mathrm{~K}^{-1}\right.$ at $10 \mathrm{~K}$ ), but its optical absorption is high (about $90 \mathrm{ppm} \mathrm{cm}^{-1}$ [85]), constraining the interferometer design and limiting the future light power increase in the main Fabry-Perot cavities. Silicon shows a similar thermal conductivity $\left(1200 \mathrm{~W} \mathrm{~m}^{-1} \mathrm{~K}^{-1}\right.$ at $12.5 \mathrm{~K})$, but it is transparent only at a longer wavelength $(\lambda \gtrsim 1500 \mathrm{~nm})$, where it shows an incredibly low absorption (about $3 \times 10^{-8} \mathrm{~cm}^{-1}$ at $1445 \mathrm{~nm}$ [86]), which requires to reconsider all optical and electro-optical component choices in the interferometer.

To solve the coating problem, either a new high refraction index material with a low dissipation at cryogenic temperature, suitable to realize the needed high reflectivity coating, is found or a completely different solution must be developed. For example, a promising R\&D activity is addressed to produce high reflectivity mirrors with just one dissipative layer of dielectric coating material on the substrate [87] or even without any additional layer, realizing the so-called resonant waveguide grating [88] by nano-structuring the surface of the silicon substrate. Other promising R\&D activities, here not discussed because of the lack of space availability, are addressed to realize the high finesse Fabry-Perot cavities, needed in the future GW detectors, minimizing the dissipating coating layers (replacing the end mirror of the long Fabry-Perot cavity with an additional anti-resonant cavity, the so-called Khalili cavity [89]), or eliminating them (using the total internal reflection phenomenon through the Brewster's angle reflectors [90] or using the corner reflectors [91]).

The coupling of the mirror surface vibration, induced by the various thermal noise sources, with the GW detector sensitivity is affected by the beam size in the Fabry-Perot cavities (larger is better). Because of the finite size of the substrates, the requirements in terms of diffraction losses and in terms of optical stability of the Fabry-Perot cavities, the beam size cannot grow infinitely and some other technique must be investigated. The potential in terms of thermal noise reduction of Fabry-Perot cavities with resonant beams with a flatter radial distribution of the light intensity [92] is well known; some attention has been drawn toward the possibility of using Laguerre-Gauss modes of orders higher than $(0,0)$ resonating in the Fabry-Perot cavities [93, 94]. According to the most recent results [95], in the case of a cryogenic interferometer $(10 \mathrm{~K})$ adopting a silicon mirror with a diameter of $62 \mathrm{~cm}$ and a thickness of $30 \mathrm{~cm}$, resonating a $(3,3)$ Laguerre-Gauss mode with a beam waist radius of $7.2 \mathrm{~cm}$ it is 
possible to suppress the cumulative mirror thermal noise by a factor 1.71 with respect to a Gaussian beam with a waist size of $11.9 \mathrm{~cm}$ (beam sizes selected to give diffraction losses of $1 \mathrm{ppm})$. These numbers show that it is important to push the development of such techniques for minimizing the thermal noise contributions to the overall noise budget.

\subsection{Quantum noise}

3.3.1. The origin of quantum noise. Quantum noise in interferometric gravitational wave detectors arises from the quantization of the electromagnetic field. All first-generation (initial) and second-generation (advanced) interferometric gravitational wave detectors are based on the Michelson principle. In all of them, the operating point is chosen such that all field components symmetrically present (in amplitude and phase) in both interferometer arms (like the laser carrier light), returning from both arms, constructively interfere toward the input (symmetric) port and destructively interfere toward the output (asymmetric) port. Asymmetric field components, i.e. introduced by a GW passing through the interferometer, show up at the asymmetric, output port. Due to phase relations (resulting from energy conservation) at the beam splitter [96], fields entering the interferometer from the output port get split into the two interferometer arms with a phase shift of $\pm \pi$, w.r.t. the light entering from the symmetric port and cause asymmetric field components in the interferometer arms, which, returning to the asymmetric port, cannot be distinguished from a GW signal. Hence it is mostly fluctuating fields entering the output port that we have to worry about. According to quantum mechanics, the lowest energy state of the quantized electromagnetic field (also called the zero-point field or vacuum state) still has energy fluctuations of $\hbar \omega / 2$ per mode of the field, distributed over both quadratures, amplitude and phase. The level of fluctuations can be traded between the quadratures as only the product is limited by Heisenberg's uncertainty principle. These fluctuations can enter the interferometer through the output port, asymmetrically add to the light field in the interferometer arms and cause noise inside the interferometer and in the output field: the quantum noise. A comprehensive description of the quantum noise and means to fight it can be found in [97] and references cited therein. The resulting strain noise spectral density for a simple Michelson interferometer is given by

$$
S_{h}=\left(\frac{1}{\kappa}+\kappa\right) \frac{h_{\mathrm{SQL}}^{2}}{2} \quad \text { with } \quad \kappa=\frac{4 I_{0} \omega_{0}}{c^{2} \mathrm{M} \Omega^{2}} \quad \text { and } \quad h_{\mathrm{SQL}}=\sqrt{\frac{4 \hbar}{M \Omega^{2} L^{2}}},
$$

where $\omega_{0}$ is the angular frequency of the laser light, $\Omega$ is the gravitational wave signal frequency, $L$ is the arm length, $c$ is the speed of light, $M$ is the mirror masses and $I_{0}$ is the laser power. The quantum noise usually shows up in two forms: shot noise and radiation pressure noise. Shot noise is the intensity quantum noise on the photo detector. Radiation pressure noise results from the impulse transfer of the photons upon reflection from a test mass. The (differential) quantum fluctuations in the power of the beams impinging on the mirrors lead to real mirror displacements having the same effect as that of a gravitational wave. In equation (1) the $1 / \kappa$ term represents the shot noise at the readout while the $\kappa$ term stands for the radiation pressure effect. The optimal sensitivity that can be reached for a given frequency by trading shot noise versus radiation pressure by choosing the best light power is called the standard quantum limit (SQL).

The interferometric measurement of gravitational waves relies on the conversion of a differential length change of the interferometer arms into a power change of the light at the output of the interferometer. Phase noise of the vacuum fluctuations which can enter the interferometer through the output port gets converted into intensity fluctuations of the light returning to the output port, which is then measured by the photo-detector. Without 
the vacuum fluctuations from the output port the signal from the interferometer would show only negligible shot noise on the output beam. While the amount of the vacuum fluctuations entering the interferometer through the output port is constant, the amount of carrier light in the interferometer arms can be changed. This results in a shot noise level on the photodetector that is proportional to the amplitude of the light leaving the interferometer output. The gravitational-wave signal on the other hand is proportional to the light power inside the interferometer, which translates into a proportionality of the light power on the photo-detector, as long as no other power-dependent processes introduce additional noise. Hence the SNR scales with the square root of the light power in the interferometer.

3.3.2. Increasing the light power. So one solution for improving the sensitivity (for frequencies where shot noise dominates) is to increase the light power inside the interferometer arms. High power in the interferometer arms is achieved by three different means:

- high power lasers

- power recycling

- arm cavities.

High power lasers. The most obvious way to increase the light power inside the interferometer is to use a powerful laser. All first- and second-generation interferometric GW detectors use lasers with a wavelength of $1064 \mathrm{~nm}$. During the construction and operation of the first generation, the available laser power increased from about 10-20 W in the first generation to $200 \mathrm{~W}$ available for the second generation. According to current plans, solid state $1 \mathrm{~kW}$ lasers will be available for the third generation. The use of high laser power gives rise to thermal problems with the light absorbed in auxiliary optics like modulators, Faraday rotators or polarizers. The absorbed light will lead to a local temperature increase which through the temperature dependence of the refractive index will lead to thermal lensing. This in turn can lead to the reduced quality of mode matching to the optical resonators. Especially the main optics in the interferometer arms will show strong thermal lensing effects as they are exposed to light power levels in the MW range in future detector generations. Although the absorption levels of fused silica have been cut down in the past to a level of $0.25 \mathrm{ppm} \mathrm{cm}^{-1}$ for bulk absorption and to the sub-ppm level for coating absorption, thermal lensing will still pose a problem and elaborated thermal compensation systems are needed to compensate the effect [98].

The need to achieve low absorption combined with good mechanical performance at low temperatures (see section 3.2.2) will require to select a new material for the test masses. The physical properties of silicon make it a good candidate. High power fiber lasers are currently being developed but have not yet reached the performance level of solid state lasers. A current overview of available laser sources and prospects for the third generation of interferometric gravitational wave detectors is given in [99].

Power recycling. The carrier light and all common fluctuations returning from the interferometer arms constructively interfere toward the input port where they can be reflected with a mirror located in the input, called the power recycling mirror. The interferometer together with this mirror then forms an optical resonator where the light power can resonantly be enhanced. This technique is called power recycling [100] and essentially has the same effect as directly increasing the laser power injected into the interferometer. All detectors of the first and second generation use and will use power recycling. With a laser power of about $200 \mathrm{~W}$, the second generation will reach power levels inside the arm cavities of around 
Table 2. Typical light powers and mirror masses for different generations of GW interferometers.

\begin{tabular}{lcrr}
\hline Detector generation & \multicolumn{1}{l}{ First } & Second & \multicolumn{1}{c}{ Third } \\
\hline Laser power & $10 \mathrm{~W}$ & $200 \mathrm{~W}$ & $1 \mathrm{~kW}$ \\
Light power @ beam splitter & $200 \mathrm{~W}$ & $2 \mathrm{~kW}$ & $20 \mathrm{~kW}$ \\
Light power inside ifo arms & $15 \mathrm{~kW}$ & $800 \mathrm{~kW}$ & $3 \mathrm{MW}$ \\
Mirror mass & $10 \mathrm{~kg}$ & $40 \mathrm{~kg}$ & $200 \mathrm{~kg}$ \\
\hline
\end{tabular}

$800 \mathrm{~kW}$. The third generation is aiming at a light power in the interferometer arms in the few megawatt range. With the promising prospect of $1 \mathrm{~kW}$ high-power lasers available for the third generation there will be no need to increase the power recycling factor beyond what is foreseen in the second generation.

Arm cavities. Placing Fabry-Perot cavities in the interferometer arms resonantly enhances the light in the individual arms and with it the signals created by the GW. The amount of power enhancement is limited by the fact that the linewidth of the optical resonator decreases with increasing build-up until relevant parts of the frequency spectrum fall outside of the resonance of the cavity and the GW signals do no longer get simultaneously enhanced with the carrier light. The low transmission of the input mirror then causes a reduction of signal amplitude for high frequency signals at the output port, i.e. a reduced sensitivity. The possible power enhancement in the interferometer arms is hence a compromise between a low relative shot noise level and bandwidth.

Approximate numbers for power requirements for the first to the third generation are given in the upper part of table 2.

3.3.3. Signal recycling. The second generation of interferometric gravitational-wave detectors (in this sense treating GEO600 as a second-generation instrument) uses an additional technique to lower the relative shot noise: signal recycling [101]. The signals being generated by the GW (with a phase difference of $\pi$ ) in the interferometer arms constructively interfere toward the output port. If these signals are sent back toward the interferometer with a recycling mirror, they get reflected back to the output port by the interferometer, similar to the laser power being reflected back to the input port. Together with the interferometer, this mirror forms an optical resonator, the signal recycling cavity. By microscopic adjustment of the position of the signal recycling mirror, the resonance of this cavity can be tuned to any desired frequency. Depending on this tuning, the bandwidth of the interferometer can either be narrowed (called signal recycling in the case where the carrier frequency is close to a resonance) or widened (called resonant sideband extraction in the anti-resonant case). The tuning changes the resonance conditions of the fields in the signal recycling cavity and with it the phase relation between the signal sidebands and the carrier at the output port and inside the interferometer arms. Signal recycling enhances the GW signal being generated inside the optical cavity but does not enhance the fluctuations entering the cavity from the output port.

Besides the optical resonance, detuned signal recycling produces another opto-mechanical resonance, typically at lower frequencies (see figure 1), which gives an improvement in sensitivity and, through the correlations that it introduces between amplitude and phase fluctuations, even allows to beat the standard quantum limit [104, 105]. Already in the advanced detectors this additional optomechanical resonance lets the quantum noise fall below the standard quantum limit, as can be seen in figure 1 . 
3.3.4. Squeezing. As indicated above, the fluctuations in the different quadratures of the field entering the output port can be traded against each other, still fulfilling the requirements of Heisenberg's uncertainty relation. If such a squeezed state, where the phase noise is lowered at the cost of the amplitude noise, is injected into the output port, the shot noise of the light registered by the photo-detector will be lowered. Squeezed light sources at $1064 \mathrm{~nm}$ were tested in many table-top experiments and at the suspended $40 \mathrm{~m}$-prototype at the California Institute of Technology [106-108]. Only recently squeezed shot-noise in the audio detection band of gravitational wave detectors was demonstrated [109, 110], a detector compatible control scheme was developed [111] and the strongest squeezing effect was ever observed [112, 113]. At the same time as the shot noise is reduced, the radiation pressure contribution will increase. By appropriately rotating the phase angle of the squeezing as a function of frequency, which can be achieved by sending the squeezed state through filter cavities [114, 102, 103], the shot noise (at high frequencies) and the radiation pressure noise (at low frequencies) can be simultaneously reduced. In case of using squeezing together with a detuned interferometer (i.e. not tuned to zero frequency, which means that the laser carrier frequency is not resonant in the signal recycling cavity), the rotation of the squeezing ellipse with respect to the light coming from the interferometer (due to the dispersion of the Signal Recycling cavity) causes an increased amount of quantum noise outside of the signal recycling resonance due to the anti-squeezed quadrature of the injected squeezing [105]. Instead of filtering the squeezed state similar to fighting the radiation pressure noise, as mentioned above, this effect can be avoided by using twin signal recycling [116]. In addition, twin signal recycling resonantly enhances both signal sidebands, at positive and negative frequencies.

Squeezing is already being implemented in the current generation of interferometric gravitational-wave detectors in the case of GEO-HF, the upgrade of GEO600 [115], aiming at a squeezing level of $6 \mathrm{~dB}$ over the entire frequency range of interest. $6 \mathrm{~dB}$ effective squeezing at the photo-detector lowers the shot noise by a factor of 2 corresponding to a light power increase of a factor of 4 . An overall gain from squeezing of about $10 \mathrm{~dB}$, i.e. a factor of 3 in noise amplitude spectral density, for the third generation presently seems realistic. Squeezed light injection may also be tested at one of the LIGO interferometers within the next years.

3.3.5. Radiation pressure noise. Whereas increasing the power in the interferometer lowers the relative readout noise, i.e. the shot noise, it increases the radiation pressure noise. The impulse transfer of the photons onto the mirrors upon reflection causes a force acting onto the mirrors, the radiation pressure $p=2 I / c$, where $I$ is the light power and $c$ the speed of light. The amplitude fluctuations on the light this way get converted into mirror motion causing the so-called radiation pressure noise. The mechanical susceptibility of a free mass (or a pendulum mass well above its resonance frequency) to a force is $(M \Omega)^{-2}$, were $M$ is the mass of the test mirror and $\Omega$ the Fourier frequency of interest. Radiation pressure therefore is more important at low frequencies, which also shows in equation 1 . The worsening of the quantum noise at frequencies below about $20 \mathrm{~Hz}$ shown in figure 1 is due to this effect.

Increasing the mirror mass decreases the mechanical susceptibility and hence cuts down the effect of radiation pressure on test mass movement. Consequently the mirror mass will increase from the first to the second generation as indicated in table 2.

Reducing the differential amplitude fluctuations in the interferometer arms by using squeezed states replacing the vacuum fluctuations entering the output port can further lower the radiation pressure effect.

3.3.6. Parametric instabilities. The high laser power, stored in the main Fabry-Perot cavities, could bring another crucial problem: the parametric instabilities [117]. In this phenomenon, 
the optical modes of the Fabry-Perot cavities couple with the acoustic models of the mirrors spoiling the cavity's performance, up to the unlock of the cavity. There are studies evaluating the effect on the advanced interferometers [118, 119] and on LCGT [120].

The relevance of the parametric instabilities issue strongly depends on the selected design options and it has been computed [121] that the number of the instable modes of the ET cavity is a few hundred times larger than that of the LCGT cavity (where the problem is negligible) and it is probable that a method to suppress the parametric instabilities is necessary (i.e. introducing a localized mechanical dissipation in the mirror's substrate, by applying a dissipative coating on the barrel surface), but a dedicated R\&D activity is needed.

\section{Scenarios for the third-generation GW observatories}

In this section, we will describe a possible evolution scenario that should lead us to the third generation of GW detectors. We will describe the most probable options based on currently known technologies. For this reason, we will neglect (although they may be scientifically interesting) some new technical options or some new solutions such as atom-interferometers [122].

\subsection{Single detector or multi-detector observatory}

As described in the previous section, to realize a third-generation GW detector, the technologies currently operative in the initial and planned for the advanced detectors must be stressed and new solutions must be adopted. Although the R\&D advances may lead to a completely different result, a third-generation GW detector could be based on the following technologies:

- long arms, probably about $10 \mathrm{~km}$ long, to enhance the sensitivity to the dimensionless spacetime strain $h$;

- underground site, to suppress the seismic and gravity gradient noises;

- long seismic filtering chains, to push the low frequency limit toward $1 \mathrm{~Hz}$;

- cryogenic test masses, to suppress suspension and mirror thermal noises;

- large and flat beams, to suppress thermal noise and mitigate the mirror thermal lensing;

- high power laser (about $1 \mathrm{~kW}$ ), high finesse Fabry-Perot cavities, high power recycling factor, signal recycling and squeezed light state injection, to suppress the quantum (shot) noise;

- heavy test masses and filtered squeezed state injection, to suppress radiation pressure noise.

A first evaluation of the potential of a similar detector has been performed [10] within the ET project, considering only conventional technologies. Targeting for a wide-band detector, the sensitivity (named ET-B) of an underground, long suspension, cryogenic, signal and power recycled single Fabry-Perot enhanced Michelson detector has been evaluated (see table 1 in [10]) and the resulting sensitivity is plotted in figure 2. In this evaluation, the crosscompatibility between the different technologies has been neglected, but the technological difficulties are evident. For example, the need of high power in the Fabry-Perot cavities (about $3 \mathrm{MW}$ ) conflicts with the requirement of a cryogenic suspension optimized for thermal noise, because of the opposite geometrical constraints given by the optimization of heat removal and the optimization of the geometrical dilution factor. Therefore, the option to realize a wide-band third-generation observatory, combining two (or more) detectors, specialized on different frequency bands, has been evaluated in [123]. Here the output of a low-frequencyspecialized detector is combined with the output of a high-frequency machine. The former 


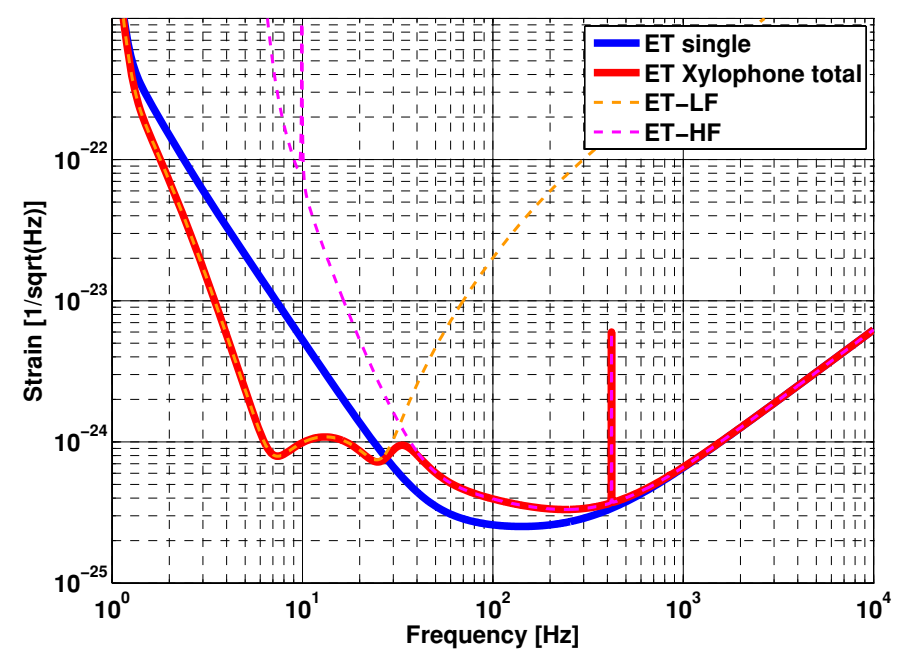

Figure 5. Sensitivity of a third-generation GW observatory implemented by two frequencyspecialized (LF and HF) detectors (xylophone topology [123], curve so-called 'ET-C'), with respect to a single wide frequency range interferometer ET implementation.

one could be a cryogenic interferometer at an underground site, with long suspensions, but moderate optical power, whereas the high frequency interferometer could essentially be a long arm advanced detector, implementing squeezed light states, a very high power laser and large test masses. This so-called xylophone philosophy [124, 125] decouples the technological requirements of a high power interferometer from the requirements of a cryogenic detector; obviously, the capability to tune the sensitivities of the two (or more) detectors constituting the observatory permit to better fit the sensitivity of such an observatory to the wanted sources, but it is worth to stress the fact that the main objective of the xylophone strategy is to avoid the technical difficulties that could make the sensitivity 'ET-B' of figure 2 an impossible target for a single wide-range interferometer implementation. A possible realization of such a xylophone strategy, evaluated in [123] for the ET design study, is plotted in figure 5.

\subsection{Detector geometry}

All the currently active GW detectors are L-shaped, with orthogonal arms; although this geometry maximizes the sensitivity of the single detector with respect to the arm length, other geometries are possible. In particular, triangular-shaped detectors have been proposed in the past $[126,127]$ and also the LISA geometry is triangular. A detailed analysis of the benefits and drawbacks of a triangular-shaped third-generation GW observatory is described in [128] and here we report only the conclusions.

Co-located interferometers could be extremely useful to extract additional information from the GW observation; for example, two L-shaped detectors, forming a $45^{\circ}$ angle, could fully resolve the two polarization amplitudes of the incoming wave. Three colocated interferometers, rotated by an arbitrary angle, through the virtual interferometry [129] technique, could do the same, supplying additional benefits like null-stream channels and redundancy. Obviously in an underground site, the realization of a similar cluster of orthogonal L-shaped detectors is practically impossible, due to the huge cost of the infrastructures (several tunnels to accommodate the arms, several caverns to realize the central and end stations). If 
the angle between the two arms of each detector is reduced to $60^{\circ}$, three detectors can be accommodated in a triangular-shaped underground site, minimizing the total length of tunnels, probably the number of caverns and recovering a sensitivity equivalent to two sets of orthogonal L-shaped double detectors, rotated by $45^{\circ}$ (see figure B1 of [128]).

For these reasons and because of the relevant role that the cost of the infrastructures will play in a third-generation GW underground observatory, the selection of the geometry will probably be driven by the selection of the site and not vice versa: if a site that can accommodate a triangular observatory is found, the triple co-located interferometers will be the best choice, otherwise the two sets of orthogonal L-shaped double detectors will be more appealing.

\subsection{Network of detectors}

So far we assumed that a single site facility forms the third-generation observatory. Depending on the astrophysical goals, the directionality of a detector could be of crucial importance, playing a role even larger than the sensitivity. This can be the case if a GW source needs to be spotted in a multitude of electromagnetic sources.

Currently a network of at least three well-separated detectors is needed to reconstruct the direction in the sky of a coalescing binary star system, the most promising GW source. In section 2.1, it has been anticipated that, thanks to the improved sensitivity, a third-generation GW observatory could gain a lot in directionality, using the additional information embedded in the full-PN approximation. In principle, two detectors at the same site could fully resolve the GW source. In practice, because of the limited accuracy, it may be advantageous to have two or more detection facilities spaced as widely as possible. Although only one thirdgeneration project is being studied now, there is hope that other projects, e.g. a LIGO successor, will follow, resulting in a worldwide network of third-generation detectors forming one big observatory. Using two distant detectors, the five parameters needed to reconstruct the source direction can be extracted from the four amplitudes from two sites and the single time delay.

For the detection of the stochastic GW background, multiple co-located detectors would be best suited. The distance should be big enough to rule out common environmental noise sources but close enough that stochastic background noise still shows up coherently in the detectors.

\subsection{Timelines}

The evolution to the third generation of GW detectors has been, is and will be a long path. Currently the main effort is made in Europe and only the European scenario will be depicted. After a series of preliminary activities supported by the European Commission within the Framework Programme 6 (FP6), a conceptual design study is currently funded under FP7: the Einstein Telescope design study [9]. This proposal was approved and funded for 3 years, starting from May 2008. The major goal of the ET project is to deliver a conceptual design for such a facility, investigating the technological feasibility, the science targets, the site requirements and prepare a costing draft for the infrastructure.

Although the efforts and the attention of the worldwide GW scientific community are currently focused on the realization of the advanced detectors (Advanced LIGO, Advanced Virgo and LCGT), the activities devoted to the third generation must continue with increasing speed. In figure 6, the expected evolution of the GW detectors in the World is shown. The last line of the table shows the long path still in front of the European project ET. After the current conceptual design study phase, a preparatory phase is expected to be necessary to define the technological details, and the legal and organizational issues. The start of construction 


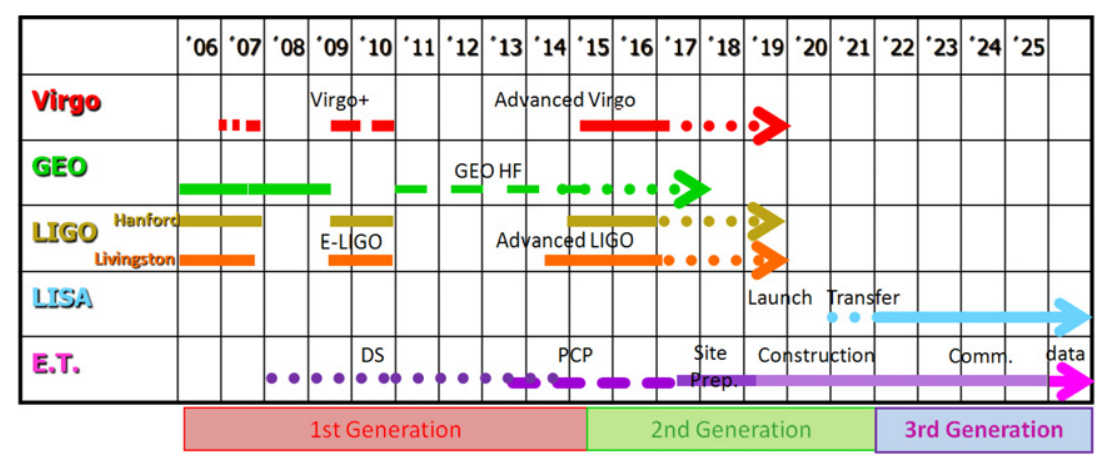

Figure 6. Roadmap for the evolution of some of the GW detectors in the World. In the last line, the expected evolution of the third-generation observatory ET (Einstein Telescope) is shown. After the current conceptual design phase (DS), and before the construction, a preparatory construction phase (PCP) is expected, where the detailed technical, legal and organization aspects of the project will be defined. In the evolution timeline of the Virgo and LIGO detectors are shown the (current) Virgo+ and E-LIGO phases, corresponding to a limited upgrade of the initial interferometers, with input laser power increased by about a factor 2-3 and other technical improvements preparatory of the 'advanced' phase. The time evolution of the Japanese interferometers (TAMA, LCGT, DECIGO) and of the other projects like ACIGA in Australia are not plotted.

(2018-2019) is expected to occur after the first detection of GWs, which is reckoned to happen within at most 1 year after the advanced detectors will have reached their nominal sensitivity. The construction and commissioning of a third-generation GW detector cannot be much shorter than what has been needed for bringing the first generation of interferometers into operation and hence a period of about 6-7 years of intense activity is deemed necessary before collecting the first science data with the Einstein Telescope ET.

\section{Acknowledgment}

This work has been performed with the support of the European Commission under the Framework Programme 7 (FP7) 'Capacities', project Einstein Telescope (ET) design study (Grant Agreement 211743) (http://www.et-gw.eu/).

\section{References}

[1] Grote H et al 2008 Class. Quantum Grav. 25114043

[2] Abbott B P et al 2009 Rep. Prog. Phys. 72076901

[3] Arai K et al 2008 J. Phys.: Conf. Ser. 120032010

[4] Acernese F et al 2008 Class. Quantum Grav. 25114045

[5] Smith J R (for the LIGO Scientific Collaboration) 2009 Class. Quantum Grav. 26114013

[6] Flaminio R et al 2005 Advanced Virgo White Paper VIRNOTDIR1390304 Acernese F et al 2009 Advanced Virgo Baseline Design VIR027A09

[7] CBC Group Rate predictions for LIGO-Virgo compact binary coalescence events https://www.lsc-group. phys.uwm.edu/ligovirgo/cbc/protected/papers/rates/rates.pdf

[8] The Virgo Collaboration Advanced Virgo Preliminary Design VIR-089A-08

[9] The Einstein Telescope design study (FP7-Capacities, Grant Agreement 211743) http://www.et-gw.eu/

[10] Hild S et al 2008 Pushing towards the ET sensitivity using conventional technology arXiv:0810.0604v2 [gr-qc]

[11] Amaro-Seoane P et al 2009 Einstein Telescope Design Study: Vision Document (ET-031-09) https://workarea.et-gw.eu/et/WG4-Astrophysics/visdoc/ 
[12] Sathyaprakash B S and Schutz B F 2009 Physics, astrophysics and cosmology with gravitational waves Living Rev. Rel. 122

[13] van Den Broeck C 2006 Class. Quantum Grav. 23 L51

[14] van Den Broeck C and Sengupta A 2007 Class. Quantum Grav. 24155

[15] Nakar E 2007 Phys. Rep. 442166

[16] Schutz B 1986 Nature 323310

[17] Sathyaprakash B S, Schutz B F and Van Den Broeck C 2009 arXiv:0906.4151

[18] Arnaud N et al 2002 Phys. Rev. D 65

[19] Nissanke S, Hughes S, Holz D E, Dalal N and Sievers J L 2009 Exploring short gamma-ray bursts as gravitationalwave standard sirens arXiv:0904.1017

[20] Blanchet L et al 1996 Class. Quantum Grav. 13575

[21] Arun K G et al 2004 Class. Quantum Grav. 213771 Arun K G et al 2005 Class. Quantum Grav. 223115 (erratum)

[22] Blanchet L et al 2002 Phys. Rev. D 65061501 Blanchet L et al 2005 Phys. Rev. D 71129902 (erratum)

[23] Blanchet L et al 2004 Phys. Rev. Lett. 93091101

[24] Choudhury T R and Padmanabhan T 2005 Astron. Astrophys. 429807 (arXiv:astro-ph/0311622)

[25] Arun K et al 2007 Phys. Rev. D 75124002

[26] Gair J R, Mandel I, Coleman Miller M and Volonteri M 2000 Exploring intermediate and massive black-hole binaries with the Einstein Telescope arXiv:0907.5450

[27] Gair J R, Sesana A and Vecchio A 2009 Probing seed black holes using future gravitational-wave detectors arXiv:0907.3292

[28] Buonanno A and Damour T 1999 Phys. Rev. D 59084006 (arXiv:gr-qc/9811091)

[29] Buonanno A and Damour T 2000 Phys. Rev. D 62064015 (arXiv:gr-qc/0001013)

[30] Damour T, Iyer B R and Sathyaprakash B S 2002 Phys. Rev. D 66027502 (arXiv:gr-qc/0207021)

[31] Damour T, Iyer B R, Jaranowski P and Sathyaprakash B S 2003 Phys. Rev. D 67064028 (arXiv:gr-qc/0211041)

[32] Damour T and Nagar A 2007 Phys. Rev. D 76064028 (arXiv:0705.2519)

[33] Buonanno A et al 2007 Phys. Rev. D 76104049 (arXiv:0706.3732)

[34] Damour T and Nagar A 2008 Phys. Rev. D 77024043 (arXiv:0711.2628)

[35] Brügmann B et al 2004 Phys. Rev. Lett. 92211101

[36] Pretorius F 2005 Phys. Rev. Lett. 95121101

[37] Campanelli M et al 2006 Phys. Rev. Lett. 96111101

[38] Baker J G et al 2007 Phys. Rev. Lett. 99181101

[39] Boyle M et al 2007 Phys. Rev. D 76124038

[40] Ajith P et al 2008 Phys. Rev. D 77104017

[41] Hannam M and Hawke I 2009 arXiv:0908.3139

[42] Weber F, Negreiros R and Rosenfield P 2007 arXiv:0705.2708

[43] Schutz B 2008 J. Phys.: Conf. Ser. 118012005

[44] Andersson N and Kokkotas K D 1998 Mon. Not. R. Astron. Soc. 2991059

[45] Ott C D 2009 Class. Quantum Grav. 26063001

[46] van den Bergh S and Tammann G A 1991 Annu. Rev. Astron. Astrophys. 29363

[47] Ando S, Beacom F and Yüksel H 2005 Phys. Rev. Lett. 95171101

[48] Braccini S et al 2005 Astropart. Phys. 23 557-65

[49] Braccini S 2009 ET internal note ET-025-09 https://workarea.et-gw.eu/et/WG5-Management/et-codifieddocuments/copy_of_et-document-codifier

[50] Abbott R et al 2002 Class. Quantum Grav. 19 1591-7

[51] Bialowons W et al 2007 Measurement of ground motion in various sites EUROTeV-Report-2007-011

[52] Uchiyama T et al 2004 Class. Quantum Grav. 21 S1161

[53] Ando M et al 2001 Phys. Rev. Lett. 863950

[54] Sato S et al 2004 Phys. Rev. D 69102005

[55] Saulson P R 1984 Phys. Rev. D 30 732-6

[56] Beccaria M et al 1998 Class. Quantum Grav. 15 3339-62

[57] Hughes S A and Thorne K S 1998 Phys. Rev. D 58122002

[58] Cella G 2009 Low Frequency Limit Talk at the Fujihara Seminar http://gw.icrr.u-tokyo.ac.jp:8888/ fujihara_seminar_presentation/presentations/Fujihara2009-Cella.pdf

[59] Cella G 2009 Gravity gradient noise: estimates and reduction strategies Talk at the 2nd ET Annual Meeting http://www.et-gw.eu/2ndgeneralworkshop 
[60] Callen H B and Welton T A 1951 Phys. Rev. 8334

[61] Kovalik J and Saulson P R 1993 Rev. Sci. Instrum. 642942

[62] Braginsky V B et al 1993 Phys. Lett. A 17582

[63] Rowan S et al 1997 Phys. Lett. A 227153

[64] Cagnoli G et al 1999 Phys. Lett. A 255 230-5

[65] Cagnoli G et al 1996 Phys. Lett. A 213 245-52

[66] Cagnoli G et al 2000 Rev. Sci. Instrum. 71 2206-10

[67] Robertson N et al 2000 Proc. of the Third E. Amaldi Conference AIP (New York) vol 313

[68] Cagnoli G et al 2000 Phys. Rev. Lett. 85 2442-5

[69] Rowan S et al 1998 Phys. Lett. A 246471478

[70] Cagnoli G et al 2002 Rev. Sci. Instrum. 73 3318-23

[71] Kuroda K et al 1999 Int. J. Mod. Phys. D 8 557-79

[72] Caparrelli S et al 2007 Report ILIAS-JR3-C1 activity http://www.ego-gw.it/ILIAS-GW/documents/ STREGAreport2007/Long reports/Report_C1_2007_D10.doc

[73] Uchiyama T et al 2000 Phys. Lett. A 273 310-5

[74] Tomaru T et al 2002 Phys. Lett. A 310 215-9

[75] Alshourbagy M et al 2006 Rev. Sci. Instrum. 77044502

[76] Reid S et al 2006 Phys. Lett. A 351 205-11

[77] Crooks D R M et al 2004 Class. Quantum Grav. 21 S1059-65

[78] Penn S D et al 2003 Class. Quantum Grav. 20 2917-28

[79] Harry G M et al 2007 Class. Quantum Grav. 24 405-15

[80] Agresti J et al 2006 Advances in thin-film coatings for optical applications III Proc. SPIE 6286628608

[81] Yamamoto K et al 2006 Phys. Rev. D 74022002

[82] Martin I et al 2008 Class. Quantum Grav. 25055005

[83] Pierro V et al 2009 Measurement of thermal noise in multilayer coatings with optimized layer thickness LIGO P0900091-x0

[84] Tomaru T et al 2002 Class. Quantum Grav. 192045

[85] Tomaru T et al 2001 Phys. Lett. A 283 80-4

[86] Green M A and Keevers M J 1995 Prog. Photovolt. Res. Appl. 3189

[87] Brückner F et al 2009 Opt. Express 17 163-9

[88] Bunkowski A et al 2006 J. Phys.: Conf. Ser. 32 333-8

[89] Khalili F Ya 2005 Phys. Lett. A 334 67-72

[90] Goßler S et al 2007 Phys. Rev. A 76053810

[91] Braginsky V B and Vyatchanin S P 2004 Phys. Lett. A 324 345-514

[92] Agresti J and Salvo R De 2005 Flat beam profile to depress thermal noise LIGO-G050041-00-Z

[93] Mours B et al 2006 Class. Quantum Grav. 23 5777-84

[94] Chelkowski S, Hild S and Freise A 2009 Phys. Rev. D 79122002

[95] Franc J et al 2009 Effect of Laguerre Gauss modes on thermal noise Talk at the ET-WP3-Meeting https://workarea.et-.gw.eu/et/WG3-.Topology/presentations/Franc-WP3-090609.ppt

[96] Zeilinger A 1981 General properties of lossless beam splitters in interferometry Am. J. Phys. 49882

[97] Corbitt T R 2008 Quantum noise and radiation pressure effects in high power optical interferometers $P h D$ Thesis MIT

[98] Vinet J-Y 2009 On Special Optical Modes and Thermal Issues in Advanced Gravitational Wave Interferometric Detectors (Living Rev. Rel. vol 12) (cited on Jan. 2010) http://www.livingreviews.org/lrr-2009-5.

[99] Mavalvala N, McClelland D E, Mueller G, Reitze D H, Schnabel R and Willke B 2009 Gen. Rel. Grav. (submitted)

[100] Schnier D et al 1997 Phys. Lett. A 225210

[101] Meers B J 1988 Phys. Rev. D 382317

[102] Corbitt T et al 2004 Optical cavities as amplitude filter of squeezed fields Phys. Rev. D 70022002

[103] Khalili F Y et al 2009 Phys. Rev. D 80042006

[104] Buonanno A and Chen Y 2003 Phys. Rev. D 67062002

[105] Buonanno A and Chen Y 2004 Phys. Rev. D 69102004

[106] McKenzie K et al 2002 Phys. Rev. Lett. 88231102

[107] Vahlbruch H et al 2005 Phys. Rev. Lett. 95

[108] Goda K et al 2008 Nat. Phys. 4472

[109] McKenzie K et al 2004 Phys. Rev. Lett. 93161105

[110] Vahlbruch H et al 2007 New J. Phys. 9371

[111] Vahlbruch H et al 2006 Phys. Rev. Lett. 97011101 
[112] Vahlbruch H et al 2008 Phys. Rev. Lett. 10033602

[113] Mehmet M et al 2009 Observation of squeezed states with strong photon number oscillations arXiv:0909.5386

[114] Kimble H J et al 2001 Phys. Rev. D 65022002

[115] Willke B et al 2006 Class. Quantum Grav. 23 S207-14

[116] Thüring A et al 2009 Opt. Lett. 34 825-6

[117] Braginsky V B et al 2001 Phys. Lett. A 287331

[118] Ju L et al 2006 Phys. Lett. A 354360

[119] Ju L et al 2006 Phys. Lett. A 355419

[120] Yamamoto K et al 2008 J. Phys.: Conf. Ser. 122012015

[121] Yamamoto K 2009 Parametric Instability of a Cavity of Einstein Telescope ET-029-09

[122] Dimopoulos S et al 2007 Gravitational Wave Detection with Atom Interferometry arXiv:0712.1250v1 [gr-qc]

[123] Hild S et al 2010 Class. Quantum Grav. 27015003

[124] Shoemaker D 2001 Presentation at Aspen Meeting http://www.ligo.caltech.edu/docs/G/G010026-00.pdf

[125] Conforto G and DeSalvo R 2004 Nucl. Instrum. Methods Phys. Res. A 518 228-32

[126] Winkler W et al 1985 Plans for a large gravitational wave antenna in Germany MPQ Report 101 Presented by A Rüdiger at the 4th Marcel Grossmann Meeting (Rome)

[127] Maischberger K et al 1985 Vorschlag zum Bau eines großen Laser Interferometers zur Messung von Gravitationswellen MPQ Report 96 (in German)

[128] Freise A et al 2009 Class. Quantum Grav. 26085012

[129] Brillet A et al 2003 Phys. Rev. D 67102006 